\title{
INFLUENCE OF SPATIAL AND TEMPORAL RESOLUTION ON TIME SERIES-BASED COASTAL SURFACE CHANGE ANALYSIS USING HOURLY TERRESTRIAL LASER SCANS
}

\author{
K. Anders ${ }^{1,2 *}$, L. Winiwarter ${ }^{1}$, H. Mara ${ }^{2,3}$, R. C. Lindenbergh ${ }^{4}$, S. E. $\operatorname{Vos}^{5}$, B. Höfle ${ }^{1,2}$ \\ ${ }^{1}$ 3DGeo Research Group, Institute of Geography, Heidelberg University, Germany - (katharina.anders, winiwarter, hoefle)@ uni- \\ heidelberg.de \\ ${ }^{2}$ Interdisciplinary Center for Scientific Computing (IWR), Heidelberg University, Germany - hubert.mara@iwr.uni-heidelberg.de \\ ${ }_{3}^{3}$ i3mainz - Institute for Spatial Information and Surveying Technology, Mainz University Of Applied Sciences, Germany \\ ${ }^{4}$ Department of Geoscience \& Remote Sensing, Delft University of Technology, The Netherlands - r.c.lindenbergh@tudelft.nl \\ ${ }^{5}$ Department of Hydraulic Engineering, Delft University of Technology, The Netherlands - s.e.vos@tudelft.nl
}

Commission II, WG II/10

KEY WORDS: LiDAR, change detection, 3D time series, spatiotemporal segmentation, 4D objects-by-change, coastal monitoring, temporal sampling, spatial sampling

\begin{abstract}
:
Near-continuously acquired terrestrial laser scanning (TLS) data contains valuable information on natural surface dynamics. An important step in geographic analyses is to detect different types of changes that can be observed in a scene. For this, spatiotemporal segmentation is a time series-based method of surface change analysis that removes the need to select analysis periods, providing socalled 4D objects-by-change (4D-OBCs). This involves higher computational effort than pairwise change detection, and efforts scale with (i) the temporal density of input data and (ii) the (variable) spatial extent of delineated changes. These two factors determine the cost and number of Dynamic Time Warping distance calculations to be performed for deriving the metric of time series similarity. We investigate how a reduction of the spatial and temporal resolution of input data influences the delineation of twelve erosion and accumulation forms, using an hourly five-month TLS time series of a sandy beach. We compare the spatial extent of 4D-OBCs obtained at reduced spatial ( $1.0 \mathrm{~m}$ to $15.0 \mathrm{~m}$ with $0.5 \mathrm{~m}$ steps) and temporal $(2 \mathrm{~h}$ to $96 \mathrm{~h}$ with $2 \mathrm{~h}$ steps) resolution to the result from highestresolution data. Many change delineations achieve acceptable performance with ranges of $\pm 10 \%$ to $\pm 100 \%$ in delineated object area, depending on the spatial extent of the respective change form. We suggest a locally adaptive approach to identify poor performance at certain resolution levels for the integration in a hierarchical approach. Consequently, the spatial delineation could be performed at high accuracy for specific target changes in a second iteration. This will allow more efficient 3D change analysis towards near-realtime, online TLS-based observation of natural surface changes.
\end{abstract}

\section{INTRODUCTION}

Time series of $3 \mathrm{D}$ point clouds are increasingly used for the observation of natural surface changes (Eitel et al., 2016). Automatic terrestrial laser scanning (TLS) from a fixed location enables to generate $3 \mathrm{D}$ time series of a topographic scene at high spatial and temporal resolution over long periods (e.g. Campos et al., 2021; O'Dea et al., 2019; Williams et al., 2018). One important reason for high-frequency acquisition is that induced surface alterations may be temporary, and hence missed or misinterpreted when using larger observation intervals (Anders et al., 2019). This applies particularly in settings where processes do not occur in a dominant and uniform direction, as with gravitational mass movements of a landslide or rockfalls (e.g., Kromer et al., 2017; Williams et al., 2018). An illustrative example of highly complex change dynamics is vegetation monitoring, where the movement of leaves and branches through wind or periodic circadian movement of trees can be captured by high-frequency TLS (Campos et al., 2021; Zlinsky et al., 2017). For the example of coastal monitoring, the observation of a sandy beach by permanent TLS enables to capture dynamic sediment transport through wind, waves, and anthropogenic modifications at centimetre scales over time spans of few hours to weeks up to seasonal or annual periods (O'Dea et al., 2019; Vos et al., 2017).
Dynamic morphologic forms such as a sand bar typically build up on a beach over several days of accumulation, and are eroded some time later, depending for example on the occurrence of high wave energy events, such as storms. In recent years, topographic data acquired by laser scanning has been increasingly used in the analysis of sandy beaches to localise morphologic forms and expand insights on sediment volume changes and transport processes (e.g. Corbì et al., 2018; de Vries et al., 2017; Le Mauff et al., 2018).

Accumulation or erosion forms can be detected in 3D time series as morphologic surface changes by performing pairwise comparisons of the topography between two epochs. Pairwise surface comparison is typically performed by differencing digital elevation models or via point cloud distance computation (Girardeau-Montaut et al., 2005; James et al., 2012; Lague et al., 2013). In landscape settings where distinct morphologic features are available, dynamics can be derived by detecting objects or characteristic features in the scene and assessing their changes in location, geometry, or size between epochs (e.g. Mayr et al., 2017; Shen et al., 2017). Features in the morphology of a scene can further be used with image correlation and range flow methods to derive 3D movement of a surface (e.g. Fey et al., 2015; Ghuffar et al., 2013).

\footnotetext{
* Corresponding author
} 
An alternative to these approaches of change analysis is required for the smooth surface morphology of sandy beach environments, where object- or feature-based methods are not suitable. Pairwise surface comparison is constrained by the need to select analysis periods, as the timing and duration of sediment transport processes is unknown.

These constraints are removed by our method of spatiotemporal segmentation, which extends change detection along the temporal domain of geographic point cloud time series (Anders et al., 2020). In this method, surface changes induced by temporary accumulation and erosion are detected in the time series at a location. Subsequently, change forms are spatially delineated with respect to their similarity of surface change history. The result of spatiotemporal segmentation are so-called $4 D$ objects-by-change (4D-OBCs) which represent the spatial extent of surface changes over the period of their existence. This method is not dependent on a selection of analysis periods or on the definition of spatial and temporal properties of changes, such as their magnitude, spatial extent, duration, or change rate (Anders et al., 2021).

The domain of surface change properties that can be observed within time series is generally determined by the resolution settings of data acquisition, according to the Nyquist-Shannon sampling theorem (Shannon, 1949). The spatial and temporal resolution of automatic TLS are best chosen to match the properties of the smallest-scale and shortest-term expected surface changes, within the limits of measurement uncertainty. In consequence, the acquisition frequency will likely be higher than necessary for many changes that occur at lower change rates or persist over longer time spans than the shortest-term changes in the scene. This abundance in data can be regarded as beneficial for exploiting the rich information on surface dynamics that acquired 3D time series contain. The drawback of incorporating the full temporal domain in change analysis, though, is a strong increase in computational cost. The balance between data volume and computational effort is gaining importance when considering online processing, which could be conducted directly on the laser scanner or on field computers.

Considering the acquisition strategy of automatic nearcontinuous TLS, 3D time series may be temporally oversampled, i.e. be much more temporally dense than required for a full spatiotemporal segmentation. A less detailed representation of change histories might hence be sufficient to extract surface changes which are covered by a high number of epochs relative to their change rate and duration.

The applied method performs spatial delineation of surface changes via region growing using time series similarity as homogeneity criterion. Time series similarity is derived as Dynamic Time Warping (DTW) distance (Berndt \& Clifford, 1994). We use the approximate FastDTW algorithm (Salvador \& Chan, 2007; Tanida, 2019), which has a linear increase in computational cost with the number of epochs. Furthermore, the number of DTW distance computations that need to be performed during region growing is determined by the number of spatial locations, which depends on the spatial extent of the change form and spatial resolution of the input data.

We therefore investigate the influence of reduced temporal and spatial resolutions of a high-frequency 3D time series on time series-based change analysis for target changes with different properties of duration, spatial extent, and magnitude. The following research questions are examined:

1) What is the influence on the spatial delineation of 4D-OBCs when reducing (i) the temporal and (ii) the spatial resolution of input data during spatiotemporal segmentation?

2) How does the influence of reduced resolutions relate to the spatial and temporal properties of detected surface changes?
If there is a relation between properties of detected changes and performance of spatiotemporal segmentation with reduced input data resolutions, it may be possible to incorporate this reduction in a hierarchical approach to analyse full 3D time series. This would allow to extract surface changes much more efficiently with similar accuracy than is currently possible.

\section{DATA AND METHODS}

We examine the influence of spatial and temporal data resolutions on change analysis by performing spatiotemporal segmentation on input data with different degrees of reduced resolutions. In the following subsections, we present the dataset of our use case (Section 2.1) and the method of spatiotemporal segmentation (Section 2.2). Subsequently, we explain our analysis approach (Section 2.3), which is schematically illustrated in Figure 1.

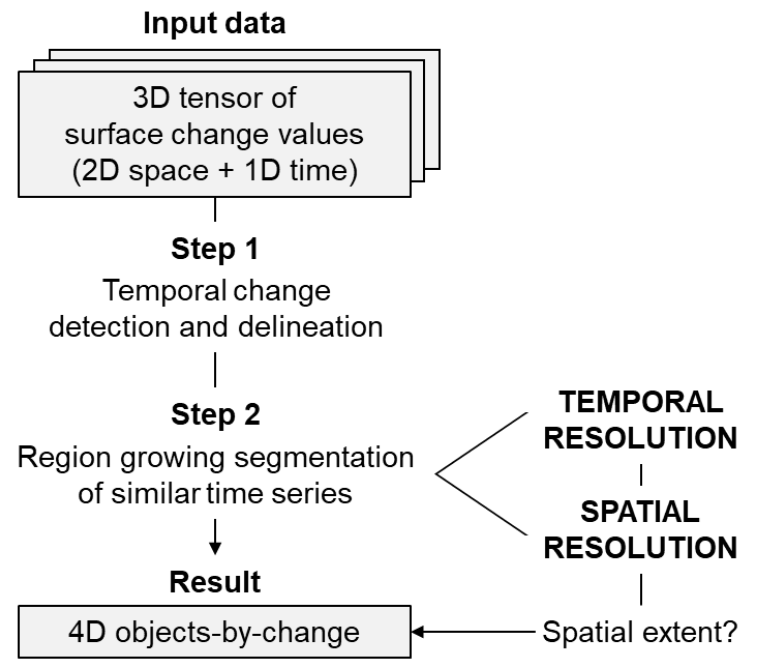

Figure 1. Experimental design to investigate the influence of reduced spatial and temporal resolutions regarding the spatial object extent resulting from region growing, compared to the results obtained at original resolution of the input data. The analysis is part of the workflow of spatiotemporal segmentation using a 3D tensor of surface change values to extract 4D objects-by-change, i.e. change forms in local areas with similar surface change history.

\subsection{Dataset}

We use a time series of TLS point clouds acquired at a beach site in The Netherlands from an elevated position on a hotel building at hourly intervals over a period of five months ( 3000 epochs of around 3 million laser points each). The TLS instrument is a Riegl VZ-2000, which scanned the underlying beach scene at ranges of $100 \mathrm{~m}$ to $600 \mathrm{~m}$ with a precision of $5 \mathrm{~mm}$ at $150 \mathrm{~m}$ range (Riegl LMS GmbH, 2015). An extensive description of the time series acquisition and TLS data properties is given by Vos et al. (2017) and Anders et al. (2019).

Within the observation period, a large number of temporary change forms were captured, which were induced by sand transport through wind and waves, as well as anthropogenic modifications. An example of an accumulation form is given with the sand bar in Figure 2. 

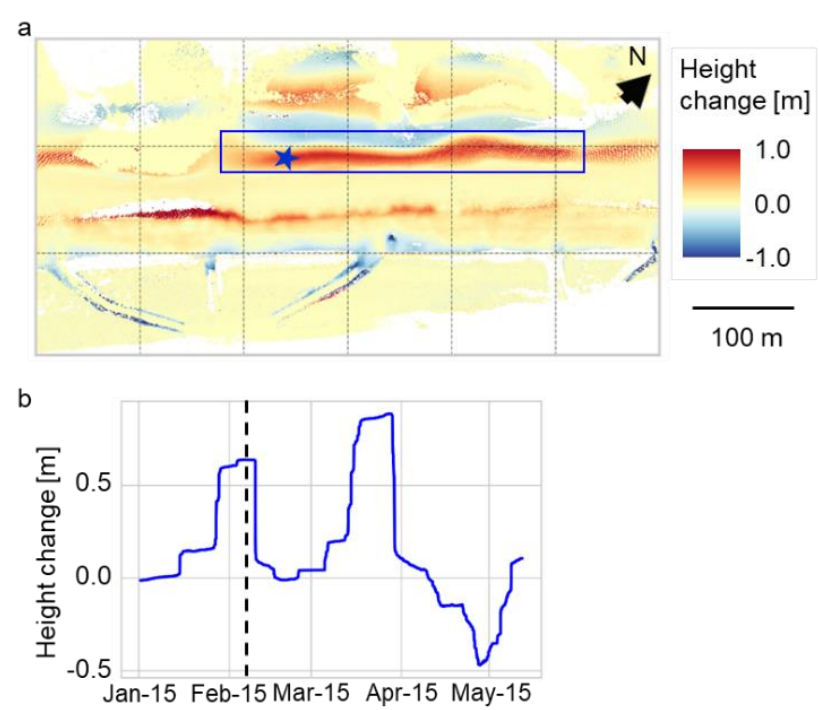

Figure 2. (a) Surface height change on the beach in a five-week period with a sand bar as notable accumulation form (blue rectangle marks bounding box). (b) History of height change at a location within the spatial extent of the sand bar (location marked by star in a). The dashed line in $b$ designates the epoch of 2017-02-20 corresponding to the surface change map in a.

Surface change is quantified for each epoch as point cloud distance to the first epoch in the time series using the M3C2 algorithm (Lague et al., 2013). Point cloud distance computation is performed for 2D locations ("core points" in the $\mathrm{M} 3 \mathrm{C} 2$ ) in a regular grid with a spacing of $0.5 \mathrm{~m}$. The $\mathrm{M} 3 \mathrm{C} 2$ assumes locally planar surfaces at each location. From this, we derive strictly vertical changes, i.e. local height increase and decrease, by setting the direction of analysis (normal vector) to be parallel to the z-axis and using a neighbourhood radius of $1.0 \mathrm{~m}$ for averaging surface positions. This creates a $3 \mathrm{D}$ tensor that contains derived surface change values at each spatial location in the scene for every epoch along the temporal domain (Fig. 2). This structured dataset provides the input for the spatiotemporal segmentation method, which is described in the subsequent section.

\subsection{Spatiotemporal Segmentation and Selected 4D-OBCs}

We use spatiotemporal segmentation as time series-based method of change analysis following Anders et al. (2021). In the first step, the method detects periods of temporary surface changes in the time series of a 2D location. In the second step, these periods of surface change at a location are grouped spatially in a region growing segmentation regarding neighbouring locations with a similar history of surface change.

For the first step of temporal change detection, change points in the time series are determined based on changes in the median of surface change values using a sliding window of one week. The end point of a temporary change form is determined by maximizing the normalized volume of surface change values along the time series from detected change points as start points. This provides the period of a detected change form at a $2 \mathrm{D}$ location. Subsequent region growing is performed using the initial 2D location as seed and time series similarity as homogeneity criterion. Time series similarity is derived as DTW distance between the time series of neighbouring locations compared to the reference time series at the seed location (Anders et al., 2020).

To account for the large ranges of magnitudes and durations in the detected changes, DTW distance values are normalized regarding the maximum possible distance to the reference time series. From this, the DTW distance threshold to constrain the region growing is determined locally adapted to each detected change. This is achieved by performing the region growing for a set of strict to loose thresholds in parallel. The final $4 \mathrm{D}-\mathrm{OBC}$ is selected at the threshold with lowest increase in segment size, which maximizes segment sizes while avoiding leakage (Anders et al., 2021).

A full spatiotemporal segmentation of the 3D time series used in our analysis yields over 2,000 4D-OBCs (cf. Anders et al., 2021). Extracted changes are temporary accumulation and erosion forms on the beach, which typically exist over periods of days to weeks. For the analysis of reduced resolutions, we select twelve 4DOBCs representing six accumulation and six erosion forms, respectively (Fig. 3). Their spatial and temporal change properties range from 6 days to 5 weeks in duration, $0.1 \mathrm{~m}$ to $2.8 \mathrm{~m}$ in magnitude, and $18 \mathrm{~m}^{2}$ to $3,000 \mathrm{~m}^{2}$ in spatial extent (Fig. 4).

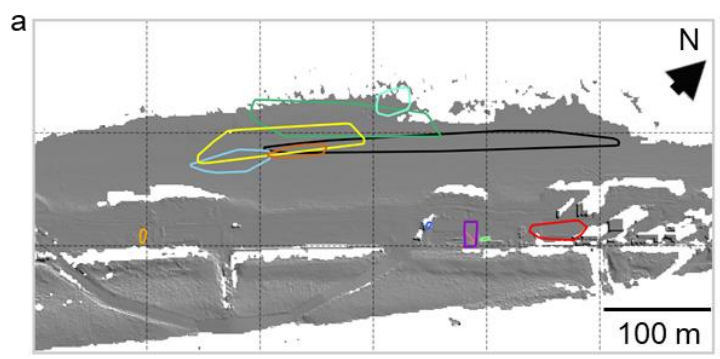

b

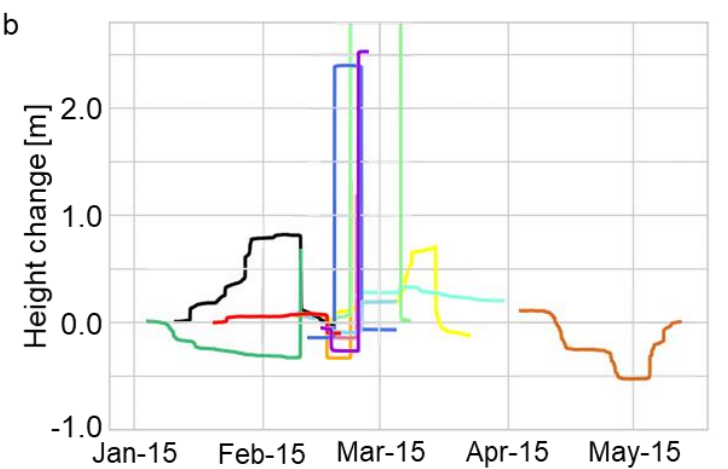

Figure 3. (a) Spatial extent in the scene and (b) change history for twelve 4D objects-by-change that are used for analysis of spatiotemporal segmentation with reduced resolutions.

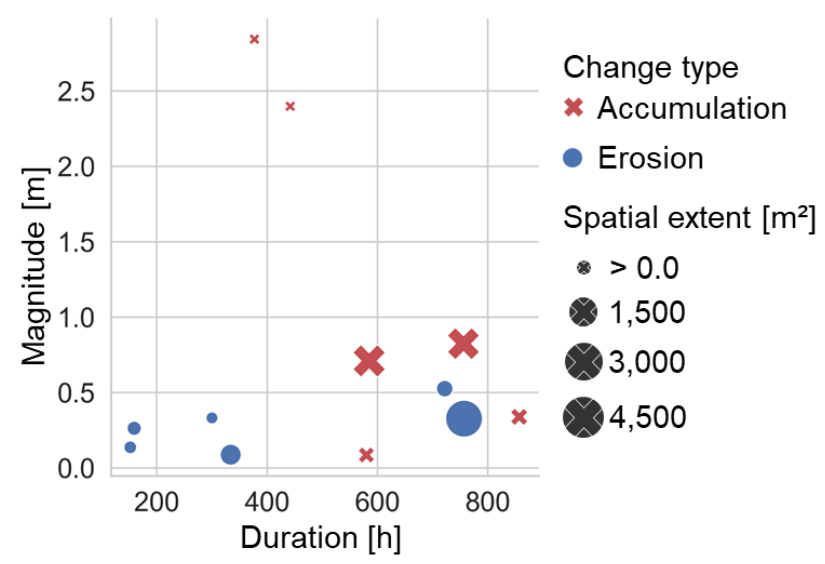

Figure 4. Spatial and temporal change properties of twelve 4D objects-by-change that are used for analysis of spatiotemporal segmentation with reduced resolutions. 


\subsection{Evaluation of Reduced Resolutions}

The influence of temporal and spatial resolutions on the extraction of $4 \mathrm{D}-\mathrm{OBCs}$ is examined by repeating the region growing on input data with iteratively increased intervals between epochs and increased spacing between 2D locations. Accordingly, only every $n^{\text {th }}$ epoch in the 3D tensor of hourly surface change is used to reduce the temporal resolution, with $n$ being multiples of the highest resolution of $1 \mathrm{~h}$, in 17 resolutions from $2 \mathrm{~h}$ to $96 \mathrm{~h}$. Only every $m^{\text {th }}$ location in $\mathrm{x}$ - and y-direction, respectively, is used to reduce the spatial resolution, with $m$ being multiples of the highest resolution of $0.5 \mathrm{~m}$, in 16 resolutions from $1.0 \mathrm{~m}$ to $15.0 \mathrm{~m}$. With reduced temporal resolution, we use the data only at highest spatial resolution, and vice versa.

The performance for all 33 resolutions of either temporally or spatially reduced input resolutions is assessed regarding the resulting spatial extent of the delineated object. The spatial extents of the 4D-OBCs acquired at the highest spatial and temporal resolution provide the reference.

Finally, the deviation of spatial extents of the derived 4D-OBCs for reduced resolutions is examined with respect to the change properties of the respective objects, namely duration and magnitude (Fig. 4). This will disclose a potential relation between properties of a detected change and required input resolutions for segmentation, which may be used to decrease the computational cost of full change analysis in 3D time series.

\section{RESULTS}

We present the results by first assessing the overall deviation of spatial extents for reduced temporal and spatial resolutions (Section 3.1). The performance of spatiotemporal segmentation regarding individual $4 \mathrm{D}-\mathrm{OBCs}$ is examined and related to their change properties in Section 3.2. In Section 3.3, we provide runtimes for the region growing depending on input data resolutions.
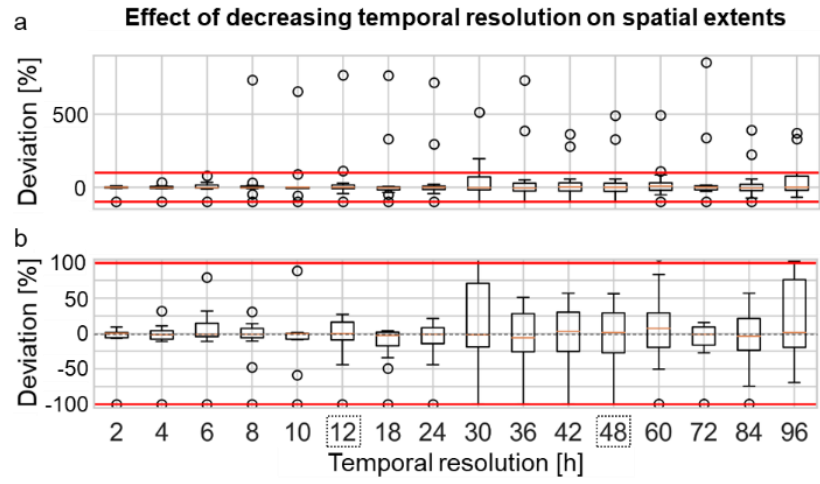

Figure 5. Deviation of spatial extent delineated by region growing for decreasing temporal resolution compared to the reference objects obtained at highest input data resolutions. (a) Full range of deviations, and (b) close-up to bounds of $100 \%$. Boxes on $\mathrm{x}$-axis labels mark changes in the reduction interval.

\subsection{Deviation of spatial extents for reduced data resolutions}

Performing the spatiotemporal segmentation of $4 \mathrm{D}-\mathrm{OBCs}$ at reduced temporal resolutions yields spatial extents with average deviations between $-6 \%$ and $+7 \%$ for resolutions from $2 \mathrm{~h}$ to $96 \mathrm{~h}$ (Fig. 5). Apart from single outliers, a notable increase in deviations begins at a temporal resolution of $30 \mathrm{~h}$, compared to the next-higher resolution of $24 \mathrm{~h}$ (Fig. 5b). This could indicate a critical level for the change forms examined in this paper, which is assessed in Section 3.3 regarding properties of individual 4DOBCs.

For reduced spatial resolutions, the variability of deviations is high for all resolution steps across the range from $1.0 \mathrm{~m}$ to $15.0 \mathrm{~m}$. Spatial extents yield average deviations between $+13 \%$ and $+260 \%$ (Fig. 6). Strong overestimations are caused by extreme increases in the delineated extent of single objects (cf. Section 3.2).

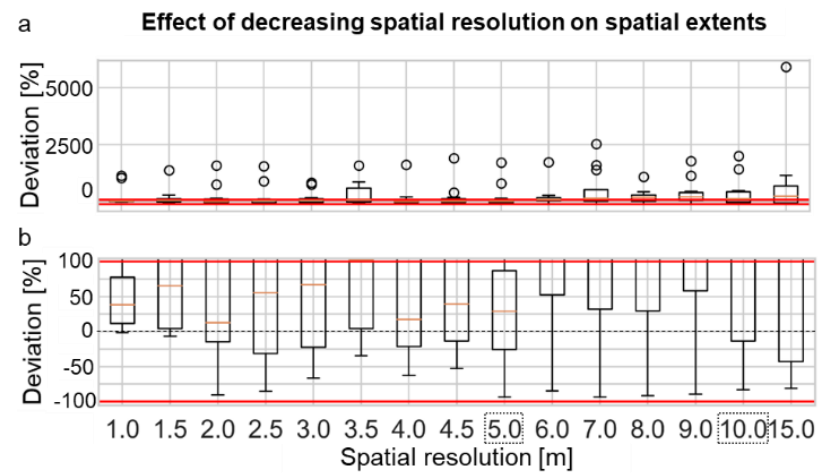

Figure 6. Deviation of spatial extent delineated by region growing for decreasing spatial resolution compared to the reference objects obtained at highest input data resolutions $(0.5 \mathrm{~m})$. (a) Full range of deviations, and (b) close-up to bounds of $100 \%$. Boxes on $\mathrm{x}$-axis labels mark changes in the reduction interval.

A remaining proportion of general over- and underestimation of spatial extents can be explained by the coarser grid resolutions of the 3D tensor. These lead to slightly larger extents at the coarser object borders with each step of reduced resolution, even if the delineation agrees well with the highest reference resolution. The same can occur for underestimation of the spatial extent, depending if the change signal is preserved during spatial subsampling of border locations to coarser resolutions. The effect is proportionally larger for change forms with smaller extent or complex shapes.

The result for an example 4D-OBC is visualized in Figure 7. This erosion form is caused by anthropogenic works, where sand was removed from rectangular patches to build up pavilions for the summer season on the beach, visible in the strong height increase following the detected change (Fig. 7b).

\subsection{Segmentation performance using reduced data resolutions in relation to individual change forms}

Reduced data resolutions have varying influence on the performance of spatial delineation for different 4D-OBCs. To assess the influence of reduced temporal resolutions, we separate the 4D-OBCs into three groups T1-T3 that show similar behaviour and scales of deviation in spatial extents (Fig. 8).

The first group (T1, Fig. 8a) yields deviations in spatial extent fluctuating over $\pm 10 \%$ to partially $\pm 100 \%$ throughout all temporal resolution steps. Deviations for these 4D-OBCs can be explained by the effect of coarser temporal data resolutions. The surface change history is either less distinct compared to the surrounding area, which may lead to more locations being segmented as similar. On the other hand, smoothing of change values in neighbouring locations compared to the distinctively detected change at the seed location will lead to fewer locations being segmented. 


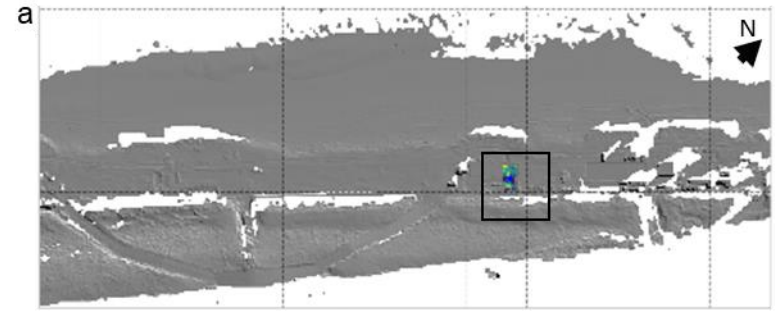

b
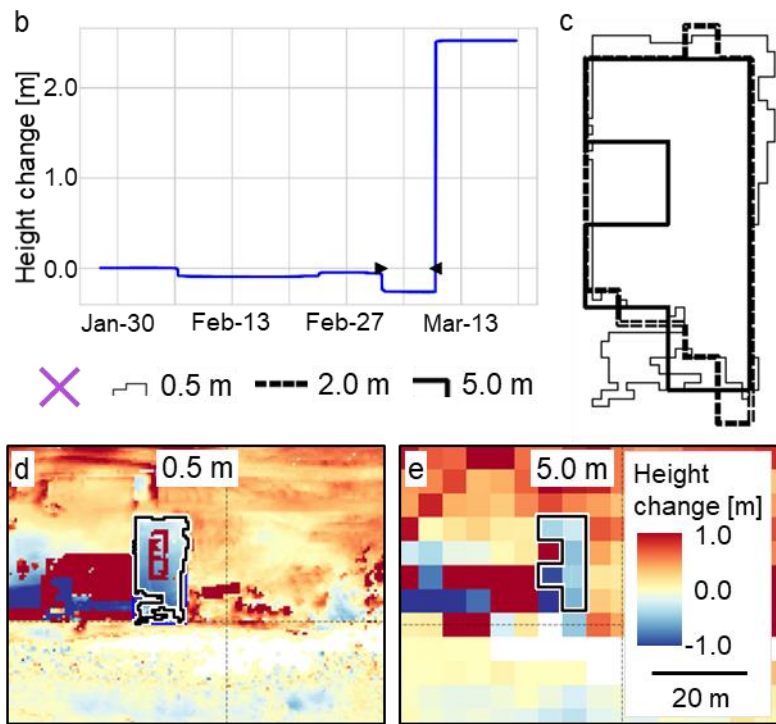

Figure 7. Results for an example 4D object-by-change using reduced spatial resolutions of $2.0 \mathrm{~m}$ and $5.0 \mathrm{~m}$. The location on the beach is depicted in the map ( $a$; rectangle marks extent of $d$ and e). The change period is marked by arrow heads in the time

series of change values at the seed location (b). Spatial delineations for different input resolutions (c) deviate from the reference by $-7 \%$ for $2.0 \mathrm{~m}$ and $-20 \%$ for $5.0 \mathrm{~m}$ resolution. The purple cross below subfigure $b$ depicts the marker used for the object in subsequent figures. Maps of height changes show the

delineated change form at reduced resolutions ( $\mathrm{d}$ and $\mathrm{e}$ ).

Group T2 (Fig. 8b) yields deviations of spatial extents below $\pm 10 \%$ down to a temporal resolution of $24 \mathrm{~h}$. At lower temporal resolutions, these $4 \mathrm{D}$-OBCs deviate up to $\pm 100 \%$, which indicates that the surface change history is not represented sufficiently in the time series anymore with respect to the surface change signals surrounding the change form. This same effect can be observed for 4D-OBCs in group T3 (Fig. 8c) with much larger negative and positive deviations (up to $1,000 \%$ ), which is visualized for an example object in Figure 9. The change form represents erosion induced by natural sediment transport followed by sudden accumulation due to anthropogenic relocation of sand volumes.

For assessing the influence of reduced spatial resolution, we separate the 4D-OBCs into another set of groups S1-S3 with similar behaviour and scales of deviation in spatial extents (Fig. 10). These show a much stronger variability of spatial extents for single objects, with deviations fluctuating between $\pm 100 \%$ even for small resolution reductions (S1 and S2, Fig. 10a and b). An enormous increase in spatial extent by up to $1,000 \%$ occurs when reducing the spatial resolution of two specific 4DOBCs (S3, Fig. 10c), which show a similarly poor performance for reduced temporal resolutions (cf. Fig. 8c).

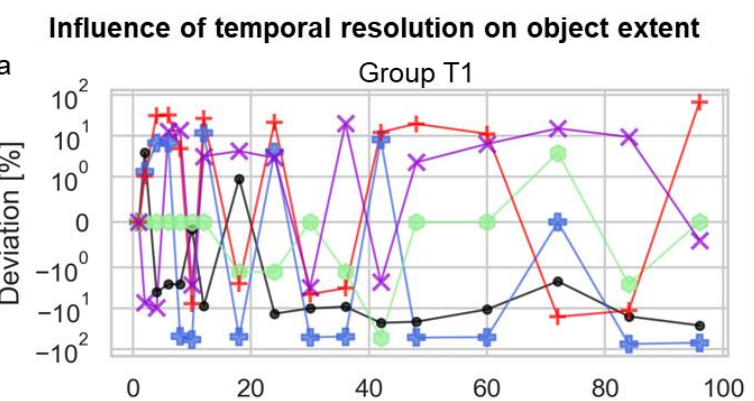

b
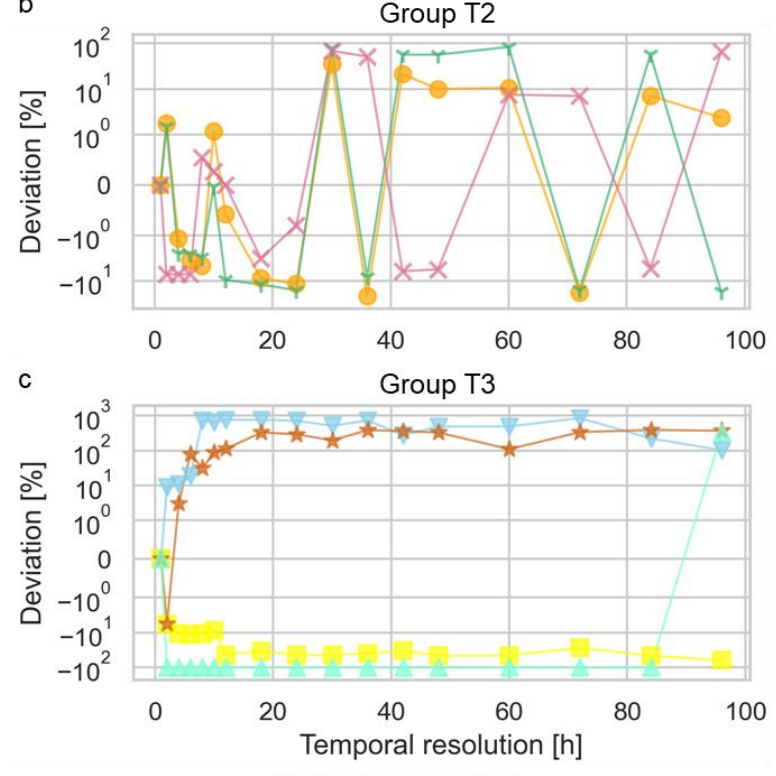

d
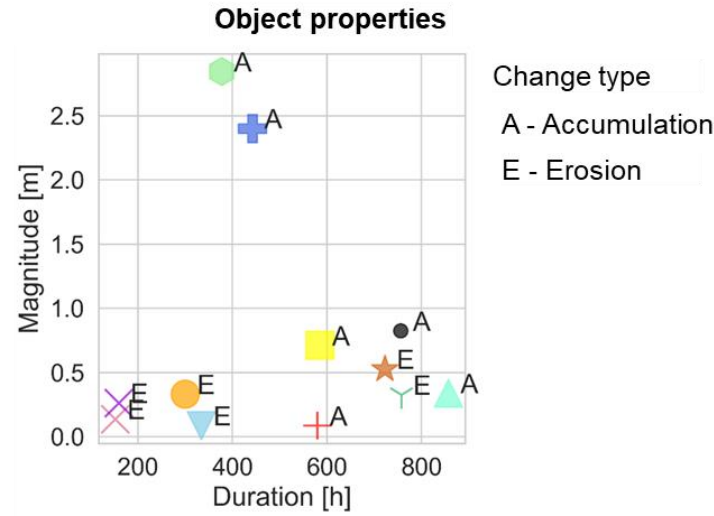

Figure 8. Relation of reduced temporal resolution to deviations in spatial extent compared to the reference objects obtained at highest input data resolutions. 4D objects-by-change are separated into three groups of similar scales and deviation behaviour (a-c). Note that the scales of y-axes are logarithmic, and only linear between $-1 \%$ and $+1 \%$. Properties of individual objects are shown with distinct markers in $\mathrm{d}$.

Comparing the results for all individual 4D-OBCs to their change properties (Fig. 8d) indicates that performance of reduced data resolutions is not solely and directly linked to the magnitude or duration of detected changes. Considering those objects with strongly over- or underestimated spatial extents, the main difference to objects with good performance is how distinct the surface change history is in relation to the surrounding surface, at each version of reduced spatial or temporal resolution. Consequently, the segmentation performance in terms of deviation of spatial extents is high for the morphometrically 
distinct changes caused by anthropogenic works even for heavily reduced resolutions (cf. Fig. 7).

For more subtle and smooth changes in the surface morphology, as is the case for gradual sediment transport, the delineation is more sensitive towards growing into adjacent locations that experience small or no surface change (cf. Fig. 9). The time series similarity will then be very high regarding the normalized DTW distance if the detected surface change at the seed location is of comparably low magnitude and long duration.
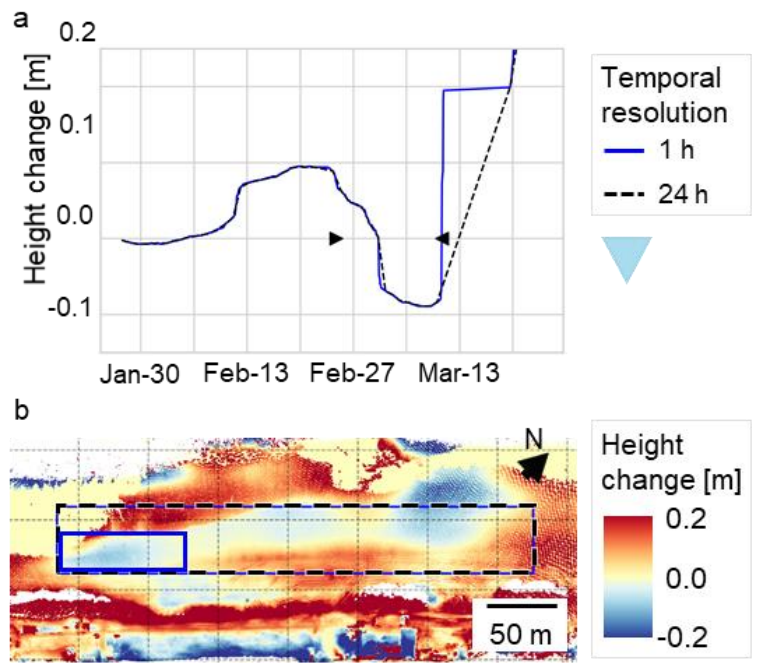

Figure 9. Results for an example 4D object-by-change using the reference and a reduced temporal resolution of $24 \mathrm{~h}$. The change period is marked by arrow heads in the time series of height changes at the seed location (a). The height change map (b) shows the bounding boxes of the delineated change form at the two temporal resolutions. An extreme increase in spatial extent $(715 \%)$ occurs at reduced resolution, as the change history of the detected change is not represented completely in the lower-resolution time series. The blue triangle below subfigure $b$ depicts the marker used for the object in other figures.

\subsection{Relation of runtimes to reduction of input data resolutions}

Regarding the objective to decrease the computational cost of time series-based change analysis of 3D time series, the relation of runtimes to reduced data resolutions (Fig. 11) fits the expectation of linear runtime reductions (Section 1). Reducing data resolutions decreases the cost and number of DTW distance calculations during region growing for reduced temporal and spatial resolution, respectively. For example, for the erosion form in Figure 9 of $780 \mathrm{~m}^{2}$ area, region growing at highest input resolution takes around 300 seconds (on a standard desktop computer). By reducing the temporal resolution to $6 \mathrm{~h}$, the runtime is reduced to $59 \mathrm{~s}$, with $+18.7 \%$ deviation in spatial extent. Occurrences of strong runtime increases at reduced resolution are caused for single $4 \mathrm{D}-\mathrm{OBCs}$ by increases in the segmented spatial extent. These increases are linked to the poor performance of spatial delineation for the respective objects (cf. Fig. 8 and 10).

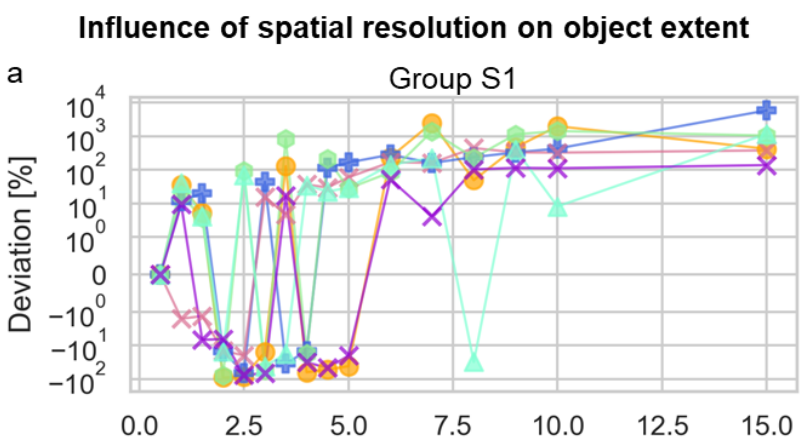

b
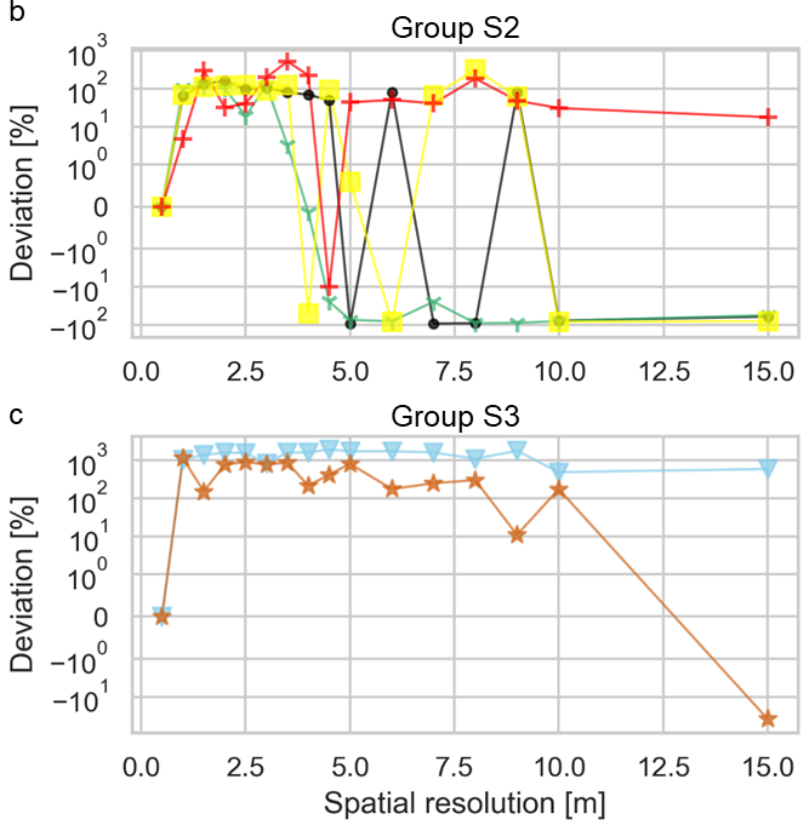

Figure 10. Relation of reduced spatial resolution to deviations in spatial extent compared to the reference objects obtained at highest input data resolutions ( $1 \mathrm{~h}$ and $0.5 \mathrm{~m})$. 4D objects-bychange are separated into three groups of similar scales and deviation behaviour (a-c) for illustration purposes. Note that the scales of y-axes are logarithmic, and only linear between -1\% and $+1 \%$. Distinct colours and markers designate individual objects, which are shown by their properties in Figure 8d.

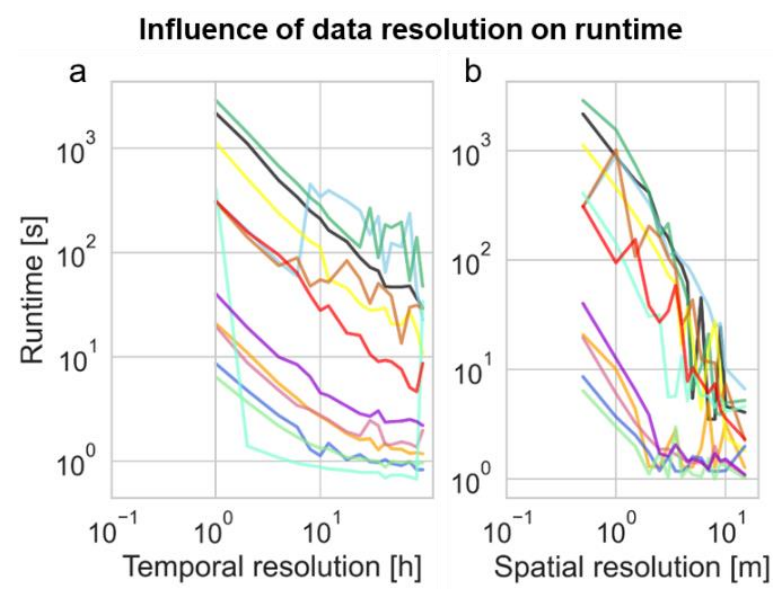

Figure 11. Relation of reduced (a) temporal and (b) spatial resolution to runtime for spatial delineation of individual 4D objects-by-change (each designated by distinct colour). Runtime values are runtimes of region growing on a standard desktop computer. 


\section{DISCUSSION}

Our results show that a reduction of input data resolutions is possible without strongly affecting the result for many of the detected changes. Deviations in spatial delineation are low when comparing the area of the objects to the highest-resolution reference with around $\pm 10 \%$ to $\pm 100 \%$, depending on the spatial extent of the detected change form. For reduced spatial resolution, deviations may be even larger at good performance, as large proportions of deviation can be attributed to border effects with more coarsely represented spatial object extents. The involved loss in spatial detail could be reduced by making use of more intelligent sampling strategies, which preserve the distinct change signals during spatial subsampling of grid locations. Preserving epochs that make surface change histories particularly distinct, such as sudden height increases or decreases, could further be integrated by thinning out time series targeted to each detected surface change. We expect that this mitigates the effect of smoothed surface change history causing the extent of a detected change form to blend into the surrounding area.

Overall, it needs to be noted that we assess the delineation performance down to very coarse resolutions ( $96 \mathrm{~h}$ and $15.0 \mathrm{~m}$ ) compared to the reference resolution $(1 \mathrm{~h}$ and $0.5 \mathrm{~m})$ and properties of the target change forms. Reducing resolutions by factor 4 (e.g. $2.0 \mathrm{~m}$ spatial resolution) to factor 12 (e.g., $12 \mathrm{~h}$ temporal resolution) would achieve an accuracy that is likely acceptable for many applications, with a deviation of $\pm 10 \%$ in spatial extent for most 4D-OBCs (cf. Fig. 8 and 10), while reducing runtimes by a factor of 10 (cf. Fig. 11).

Introducing such overall performance decrease in spatial delineation by reducing data resolutions may even be generally acceptable for the first step of change analysis, where surface changes are detected across space and time and coarsely extracted as 4D-OBCs. A more exact spatial extent can then be delineated in a second step of region growing at highest resolutions, maybe restricted to specific target changes depending on the application and research question.

A simple relation between change properties and requirements to input data resolutions does not become evident from our analysis. Nonetheless, a practical approach could make use of reduced resolutions after seed detection by performing the region growing for a set of resolutions in parallel. From our investigation, we find that there is no constant decrease of performance in spatial delineation with decreasing resolution. Many cases of poor performance of individual 4D-OBCs exhibit a high variability of deviations to the reference for iteratively decreased resolutions (cf. Fig. 8 and 10). The possibility to reduce input data resolutions with acceptable performance of results could therefore be assessed regarding changes in resulting segment sizes. Based on this information, the appropriate resolution per change form could be selected, subsequently. A similar approach was shown to perform well for determining the locally adaptive DTW distance threshold used for region growing in the method (Anders et al., 2021). When making use of parallel computing, this does not necessarily increase runtimes. Region growing of strongly oversized objects at lower resolutions takes much longer runtimes than delineating objects correctly at higher resolutions (cf. Fig. 11). In these cases, the growing can be aborted once the result of a higher resolution with smaller, more confidently delineated spatial extent is available.

\section{CONCLUSION}

In this paper, we propose the reduction of input data resolutions for time series-based change analysis using a method of spatiotemporal segmentation. Many change delineations at reduced temporal or spatial resolution achieve acceptable performance. Depending on the spatial extent and shape of the respective change form, deviations in area range from $\pm 10 \%$ to $\pm 100 \%$ compared to the reference extracted at the highest available resolution. A large variability in performance resulted particularly from reducing the spatial resolution.

The investigated 4D objects-by-change did not reveal a systematic relation of segmentation performance at reduced resolutions with respect to properties of the detected changes, such as magnitude or duration of surface change. Results rather indicate an important link to the distinctiveness of surrounding surface change histories.

Spatiotemporal segmentation enables to extract changes of variable spatial and temporal properties from $3 \mathrm{D}$ time series at a high detection rate, by incorporating the full temporal domain and using an efficient input data structure with the 3D tensor of surface change values. To integrate reduced resolutions in the method, we suggest a locally adaptive approach based on parallel computing at multiple resolutions. While decreased data resolutions in this step lead to further coarsening of the acquired 3D time series information, the original high-resolution data is still available for the interpretation of extracted surface changes and all subsequent analyses. Integrating adaptively reduced data resolutions would extend efficiency in a hierarchical approach to change analysis. In the future, this may allow to analyse 3D time series in near-realtime and to incorporate the information in online adaptive TLS-based monitoring.

\section{ACKNOWLEDGEMENTS}

This work was supported in part by the Heidelberg Graduate School of Mathematical and Computational Methods for the Sciences (HGS MathComp), founded by DFG grant GSC 220 in the German Universities Excellence Initiative. The acquisition of the TLS time series data was financed by the ERC Advanced Grant Nearshore Monitoring and Modeling (grant number 291206) and by the CoastScan project in the NWO Open Technology Programme (grant number 16352).

\section{REFERENCES}

Anders, K., Lindenbergh, R.C., Vos, S., Mara, H., de Vries, S. Höfle, B., 2019. High-Frequency 3D Geomorphic Observation Using Hourly Terrestrial Laser Scanning Data Of A Sandy Beach. ISPRS Ann. Photogramm. Remote Sens. Spatial Inf. Sci., IV-2/W5, 317-324, doi.org/10.5194/isprs-annals-IV-2-W5-3172019.

Anders, K., Winiwarter, L., Lindenbergh, R., Williams, J.G., Vos, S.E., Höfle, B., 2020. 4D objects-by-change: Spatiotemporal segmentation of geomorphic surface change from LiDAR time series. ISPRS Journal of Photogrammetry and Remote Sensing, 159, 352-363, doi.org/10.1016/j.isprsjprs.2019.11.025.

Anders, K., Winiwarter, L., Mara, H., Lindenbergh, R., Vos, S.E., Höfle, B., 2021. Fully automatic spatiotemporal segmentation of 3D LiDAR time series for the extraction of natural surface changes. ISPRS Journal of Photogrammetry and Remote Sensing, 173, 297-308, doi.org/ 10.1016/j.isprsjprs.2021.01.015.

Berndt, D. J., Clifford, J., 1994. Using Dynamic Time Warping to Find Patterns in Time Series. AAAI-94 Workshop on Knowledge Discovery in Databases, 10(16), 359-370. 
Campos, M. B., Litkey, P., Wang, Y., Chen, Y., Hyyti, H., Hyyppä, J., Puttonen, E. (2021). A Long-Term Terrestrial Laser Scanning Measurement Station to Continuously Monitor Structural and Phenological Dynamics of Boreal Forest Canopy. Frontiers in Plant Science, 11(2132), doi.org/10.3389/fpls.2020.606752.

Corbí, H., Riquelme, A., Megías-Baños, C., Abellan, A. (2018). 3-D Morphological Change Analysis of a Beach with Seagrass Berm Using a Terrestrial Laser Scanner. ISPRS International Journal of Geo-Information, 7(234), doi.org/10.3390/ijgi7070234.

de Vries, S., Verheijen, A., Hoonhout, B., Vos, S., Cohn, N., Ruggiero, P. (2017). Measured spatial variability of beach erosion due to aeolian processes. Proc. of Coastal Dynamics 2017, 71, pp. 481-491.

Eitel, J.U.H., Höfle, B., Vierling, L.A., Abellán, A., Asner, G.P., Deems, J.S., Glennie, C.L., Joerg, P.C., LeWinter, A.L., Magney, T.S., Mandlburger, G., Morton, D.C., Müller, J., Vierling, K.T., 2016. Beyond 3-D: The new spectrum of lidar applications for earth and ecological sciences. Remote Sensing of Environment, 186, 372-392, doi.org/10.1016/j.rse.2016.08.018.

Fey, C., Rutzinger, M., Wichmann, V., Prager, C., Bremer, M., Zangerl, C. (2015). Deriving 3D displacement vectors from multi-temporal airborne laser scanning data for landslide activity analyses. GIScience \& Remote Sensing, 52(4), 437-461, doi.org/10.1080/15481603.2015.1045278.

Ghuffar, S., Székely, B., Roncat, A., Pfeifer, N. (2013). Landslide Displacement Monitoring Using 3D Range Flow on Airborne and Terrestrial LiDAR Data. Remote Sensing, 5(6), 2720-2745, doi.org/10.3390/rs5062720.

Girardeau-Montaut, D., Roux, M., Marc, R., Thibault, G., 2005. Change detection on points cloud data acquired with a ground laser scanner. Proceedings of the ISPRS Workshop Laser scanning 2005, Enschede, NL, 30-35.

James, L. A., Hodgson, M. E., Ghoshal, S., Latiolais, M. M., 2012. Geomorphic change detection using historic maps and DEM differencing: The temporal dimension of geospatial analysis. Geomorphology, 137(1), 181-198, doi.org/10.1016/j.geomorph.2010.10.039.

Kromer, R.A., Abellán, A., Hutchinson, D.J., Lato, M., Chanut, M.-A., Dubois, L., Jaboyedoff, M., 2017. Automated Terrestrial Laser Scanning with Near Real-Time Change Detection Monitoring of the Séchillenne Landslide. Earth Surf. Dynam., 5, 293-310, doi.org/10.5194/esurf-5-293-2017.

Lague, D., Brodu, N., Leroux, J., 2013. Accurate 3D comparison of complex topography with terrestrial laser scanner: Application to the Rangitikei canyon (N-Z). ISPRS Journal of Photogrammetry and Remote Sensing, 82, 10-26, doi.org/10.1016/j.isprsjprs.2013.04.009.

Le Mauff, B., Juigner, M., Ba, A., Robin, M., Launeau, P., Fattal, P. (2018). Coastal monitoring solutions of the geomorphological response of beach-dune systems using multi-temporal LiDAR datasets (Vendée coast, France). Geomorphology, 304, 121-140, doi.org/10.1016/j.geomorph.2017.12.037.

Mayr, A., Rutzinger, M., Bremer, M., Oude Elberink, S., Stumpf, F., Geitner, C. (2017). Object-based classification of terrestrial laser scanning point clouds for landslide monitoring. The

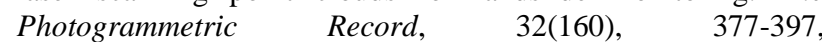
doi.org/10.1111/phor.12215.

O'Dea, A., Brodie, K. L., \& Hartzell, P. (2019). Continuous Coastal Monitoring with an Automated Terrestrial Lidar Scanner. Journal of Marine Science and Engineering, 7(2), 37, doi.org/10.3390/jmse7020037.

Riegl LMS GmbH (2015): Riegl VZ-2000 (datasheet). URL: https://kb.unavco.org/kb/assets/820/DataSheet_VZ-2000_201503-24.pdf (2021-04-12).

Salvador, S., Chan, P., 2007. Toward accurate dynamic time warping in linear time and space. Intell. Data Anal., 11(5), 561580, doi.org/10.3233/IDA-2007-11508.

Shannon, C. E. (1949). Communication in the Presence of Noise. Proceedings of the IRE, 37(1), 10-21, doi.org/10.1109/JRPROC.1949.232969.

Shen, Y., Lindenbergh, R., Wang, J. (2017). Change Analysis in Structural Laser Scanning Point Clouds: The Baseline Method. Sensors, 17(1), 25, doi.org/10.3390/s17010026.

Tanida, K., 2019. fastdtw - A Python implementation of FastDTW [version: 0.3.2], https://github.com/slaypni/fastdtw.

Vos, S., Lindenbergh, R., de Vries, S., 2017. CoastScan: Continuous Monitoring of Coastal Change using Terrestrial Laser Scanning. Proc. of Coastal Dynamics 2017, 233, $1518-1528$

Williams, J.G., Rosser, N.J., Hardy, R.J., Brain, M.J., Afana, A.A., 2018. Optimising 4-D surface change detection: an approach for capturing rockfall magnitude-frequency. Earth Surf. Dynam., 6, 101-119, doi.org/10.5194/esurf-6-101-2018.

Zlinszky, A., Molnár, B., Barfod, A. S. (2017). Not All Trees Sleep the Same - High Temporal Resolution Terrestrial Laser Scanning Shows Differences in Nocturnal Plant Movement. Frontiers in Plant Science, 8(1814). doi.org/10.3389/fpls.2017.01814. 